\title{
Cruciferous vegetables: prototypic anti-inflammatory food components
}

\author{
Herbert Tilg
}

\begin{abstract}
There is increasing evidence that food components contribute to the pathogenesis of various disorders such as inflammatory bowel diseases, atherosclerosis, cancer or type 2 diabetes. Dietary factors especially enriched in Western diet cause and promote inflammatory processes throughout the organism involving various pathways but mainly the induction of pro-inflammatory cytokines. In contrast, there is increasing evidence that certain food components such as present in cruciferous vegetables have important anti-inflammatory properties. Cruciferous vegetables contain large amounts of various indole derivatives and are able via these components to activate aryl hydrocarbon receptors (AhR). Activation of these intracellular receptors results in potent intestinal immune modulation including regulation and maintenance of intestinal intraepithelial lymphocytes and innate lymphoid cells, induction of the key barrier cytokine interleukin-22 and manipulation of the intestinal microbiota. Lack of AhR is associated with an impaired barrier function and increased intestinal vulnerability suggesting that the continuous presence of dietary AhR ligands may be of importance throughout life. Sulforaphane, an isothiocyanate compound of cruciferous vegetables, also exerts mainly anti-inflammatory properties on immune processes. Therefore, evidence is accumulating that certain food components are healthy by targeting intestinal immune responses and reshaping the microbiota.
\end{abstract}

Keywords: Anti-inflammatory; Carbazoles; Cruciferous plants; Isothiocyanates; Healthy food

\section{Introduction}

Diet is a well established risk factor for many disorders ranging from inflammatory bowel diseases (IBD), type 2 diabetes, atherosclerosis and various cancers [1]. The marked increase of many of these disorders has been paralleled in the last decades by changing habits regarding food consumption and overall industrialization. Epidemiological studies have revealed that many inflammatory conditions are associated with increased consumption of a Western diet enriched in saturated fatty acids, carbohydrates, refined grains, processed red meat and a low content of vegetables, fruits and fish. This diet is increasingly consumed worldwide and it is assumed that the spread of such dietary behaviors has contributed significantly to changing disease patterns as e.g. observed for IBD. Diets enriched in vegetables and fruits such as the Mediterranean diet have been proposed to provide health benefits

Correspondence: herbert.tilg@i-med.ac.at

Department of Internal Medicine I, Endocrinology, Gastroenterology \& Metabolism, Medical University Innsbruck, Innsbruck, Austria
[2]. Therefore, dietary components overall might have a major impact on disease pathogenesis and manifestation.

It is increasingly understood that dietary components interact with the host mainly by affecting and shaping the intestinal microbiota and immunity [3]. The interaction between food components and the gastrointestinal tract involves several components of the immune system including intestinal intraepithelial lymphocytes, innate lymphoid cells, immune mediators, epithelial cells and a heterogeneous group of immune cells including dendritic cells [1]. Molecular pathways used by both pathogens and dietary components such as the aryl hydrocarbon receptor (AhR) could be an example for understanding how diet affects and drives intestinal immunity [4-6]. The microbiota is the key factor determining development and maintenance of the intestinal and systemic immune response $[7,8]$. The diet controls the dynamics and composition of the microbiota, and the relationship diet-microbiota - immunity is essential for human development and health. The contributions of the intestinal microbiota to development of immunity 
and respective effects of dietary components on its composition are complex and beyond the scope of this review. The adage "You are what you eat" is increasingly supported by scientific evidence. This article will briefly summarize aspects of pro-and anti-inflammatory food components focusing on healthy aspects of cruciferous vegetables.

\section{Review}

\section{Inflammatory diets: potential mode of actions}

The incidence of many chronic inflammatory disorders has changed dramatically in the last three to four decades. Many of those disorders such as IBD, rheumatoid arthritis, metabolic syndrome, and atherosclerosis, are characterized by continuous low-grade systemic inflammation. In most of those diseases environmental factors such as diet seem to play a major role as genetic factors can only explain the minority of cases. Western diets may promote inflammatory processes through various mechanisms. Fatty acids support inflammation through various mechanisms, including direct actions on immune cells, toll-like receptors (TLRs), and cytokine signaling, as well by affecting intestinal permeability $[9,10]$. In healthy subjects, a high-fat Western diet results in endotoxemia and may thereby lead to low-grade systemic inflammation [11, 12]. A high fat diet (HFD) induces intestinal inflammation and the expression of tumor necrosis factor-alpha (TNF- $\alpha$ ) in the ileum in obesity, and this effect is only observed in conventionally raised specific-pathogen free mice, but not in germ-free mice [13]. As a Western diet is enriched in polyunsaturated fatty acids (PUFA), this mechanism has also been shown to contribute to intestinal inflammation especially in older mice, as demonstrated by increased influx of neutrophils and macrophages [14]. Importantly, Western diet is especially enriched in n-6 PUFA and deficient in n-3 PUFA and it is assumed that the ratio of n-6 to n-3 PUFA in Western diet is an issue but not PUFA in general. Overall it can be concluded that Western diets affect immunity, promote inflammation, and these effects importantly also include major effects on the microbiota and metabolome via many pathways. All these effects may negatively influence human health and contribute to the disease burden which is caused by consumption of an imbalanced and rather "pro-inflammatory" Western diet. A more extensive discussion of these "pro-inflammatory" aspects of diet is beyond the focus of this article.

\section{Anti-inflammatory diets: Cruciferous vegetables as prototypic proponents} Diet-derived indole derivatives as major activators of aryl hydrocarbon receptors (AhR)

A main question remains what happens in humans when they consume higher amounts of cruciferous vegetables.
Jiang and colleagues [15] have studied the effects of vegetable intake in 1005 middle-aged Chinese women. Indeed, an increased intake of cruciferous vegetables was associated with decreased serum levels of the proinflammatory cytokines IL-1 $\beta$, TNF $\alpha$ and IL-6 supporting the concept of many in vitro studies that this type of diet may have anti-inflammatory properties. As nutrition contributes substantially to disease development, it remains crucial and mandatory to better understand how diets enriched in vegetables and fruits might constitute anti-inflammatory effects. Aryl hydrocarbon receptor, a transcription factor expressed by immune cells, epithelial cells, and some tumor cells, has been identified as fundamental receptor for certain dietary components. Indeed, in the last years many exogenous and endogenous AhR ligands have been characterized-some derived from foods such as broccoli, other include phytochemicals, natural chemicals, and bacterial metabolites. Ligand binding activates the AhR, resulting in its translocation into the nucleus, where it dimerizes with the AhR nuclear translocator. This heterodimer regulates many genes that control immunity and inflammation, such as the important barrier cytokine IL-22, which has many beneficial metabolic functions [16].

Several studies published in the last few years have highlighted how dietary-derived AhR ligands affect local/ systemic immunity. Specific components of certain vegetables of the family Brassicaceae (for example, broccoli, Brussel's sprouts or cabbage) are physiologic ligands of the AhR. Li et al. [5] observed that AhR signaling maintains numbers and functions of intraepithelial lymphocytes and innate lymphoid cells, and that AhR-deficiency increased epithelial vulnerability and immune activation in mice. AhR-deficiency furthermore affected the microbiota, and decreased intestinal production of granzymes A and B, C-type lectins, and matrix metalloproteinase-7, accompanied by a 4-fold increase in the proportion of Bacteroidetes. Interestingly, absence of AhR ligands increased severity of colitis in mice; when animals were fed with diet enriched in AhR ligands, observed alterations were reversed. Kiss et al. [6] showed that activation of AhR by dietary ligands is essential for post-natal expansion of certain innate lymphoid cells and the development of intestinal lymphoid follicles. Mice deficient in AhR exhibited an impaired intestinal immunity and were highly susceptible to infection with Citrobacter rodentium. AhR ligands increased numbers of IL-22producing ROR $\gamma \mathrm{t}^{+}$intestinal lymphoid cells. These cells contribute in a major fashion to gastrointestinal innate immune functions such as the production of antimicrobial peptides and mucus and maintenance of epithelial integrity. Also other studies have shown that AhR is required for the production of IL-22, supporting the importance of this relationship [17]. The studies of Li et al. 
[5] and Kiss [6] et al. provide evidence that some sort of "dietary pattern recognition receptors" might exist that link diet with intestinal immunity and the microbiota (Table 1).

The pathway between AhR ligands and IL-22 is highly relevant in the area of intestinal immunity and metabolism. Administration of IL-22 diminished metabolic defects and restored mucosal immunity to mice on HFDs, as well as in leptin receptor-deficient $(d b / d b)$ mice [16]. Overall, dietary factors that activate the AhR have the capacity to affect expression of cytokines (particularly IL-22), synthesis of certain mucins, production of antimicrobial peptides, and consequently shape the intestinal barrier and furthermore the composition of the intestinal microbial community. Although findings of these two major studies are solely based on preclinical experimental data, they could have major clinical implications as (i) a continuous presence of beneficial dietary antigens might be needed in the intestinal tract to maintain functionality of the immune system including tolerance throughout life and (ii) if oral supplementation of such beneficial food components is interrupted such as observed in intensive care patients undergoing longterm parenteral nutrition this could severely impair local immunity, barrier function and at the end contribute to disease burden as observed in such patients.

\section{Further beneficial examples for indole derivatives}

Concentrations of carbazoles may be increased by other dietary compounds such as quercetin, resveratrol, and curcumin as demonstrated recently [18]. These compounds induce cytochrome P4501A1 in an indirect manner by inhibiting the metabolism of indole derivatives. Indole-3 carbinole (I3C) and its derivatives may also have beneficial effects on bone metabolism. An acid-condensation product of I3C, 3,3'-diindolymethane (DIM), prevents ovariectomized-induced bone loss by suppressing osteoclastic bone resorption [19]. Although not studied, it can be speculated that interference with the pro-inflammatory cytokine milieu in the bone as observed in osteoporosis might be one of those protective mechanisms. Administration of DIM also suppressed the nuclear factor kappaB (NF-kB) signaling pathway in microglia and protected cortical neurons from inflammatory toxicity [20]. When tested in mice, DIM attenuated LPS-induced brain inflammation in mouse hippocampus. Interestingly, in this model I3C did not show protective effects. When male C57BL/6 mice received a HFD and were treated intraperitoneally with $\mathrm{I} 3 \mathrm{C}$ for 12 weeks, this resulted in a profound improvement of metabolic inflammation in adipose tissue by the substantial decrease of macrophage infiltration and their cytokine production [21].

\section{Tryptophan: a dietary anti-inflammatory amino acid}

The essential amino acid tryptophan is another nutrient also found in cruciferous vegetables which shows antiinflammatory activities. Tryptophan is metabolized by the microbiota, e.g. Lactobacilli, to indole-3-aldehyde, another AhR agonist. This interaction is accompanied by induction of IL-22, which affected the microbiota, providing resistance to colonization by Candida albicans and protecting the mucosa against inflammation. This study therefore nicely highlights how another beneficial nutrient might results in gastrointestinal health again involving the microbiota and various immune pathways [22]. Tryptophan might exert anti-inflammatory effects via additional pathways such, as after conversion to kynurenine by indoleamine 2,3-dioxygenase (IDO). Both kynurenine and IDO have immunomodulatory functions that include promotion of regulatory $\mathrm{T}$ cells and

Table 1 Effects of anti-inflammatory diets on immunity

\begin{tabular}{|c|c|c|c|c|c|}
\hline \multirow[t]{5}{*}{ Anti-inflammatory } & Foods & $\begin{array}{l}\text { Microbiota- } \\
\text { dependent }\end{array}$ & Potential pathways & Effects on Immunity & References \\
\hline & $\begin{array}{l}\text { cruciferous vegetables } \\
\text { (carbazoles) }\end{array}$ & $+{ }^{a}$ & $\begin{array}{l}\text { AhR ligands } \\
\text { Suppression of NFKB }\end{array}$ & $\begin{array}{l}\text { IL-22 } \uparrow, \text { maintenance of intraepithelial } \\
\text { lymphocytes and innate lymphoid cells, } \\
\text { suppression of inflammation }\end{array}$ & {$[5,6]$} \\
\hline & $\begin{array}{l}\text { Cruciferous vegetables } \\
\text { and fish (tryptophan) }\end{array}$ & $+b$ & AhR ligands GPCRs & $\begin{array}{l}\mathrm{IL}-22 \uparrow \text {, mucosal protection from } \\
\text { inflammation }\end{array}$ & {$[5,6,17]$} \\
\hline & $\begin{array}{l}\text { cruciferous vegetables } \\
\text { (sulfarophane) }\end{array}$ & $?$ & Suppression of NFKB & $\begin{array}{l}\text { Suppression of inflammation, induction } \\
\text { of apoptosis, activation of phagocytosis }\end{array}$ & {$[23,24]$} \\
\hline & $\begin{array}{l}\text { mediterrenian diet } \\
\text { (enriched in } \omega-3 \text { fatty acids) }\end{array}$ & $?$ & Gpr120 & pro-inflammatory cytokines $\downarrow$ & {$[43]$} \\
\hline
\end{tabular}

Abbrevations

$A h R$ aryl hydrocarbon receptor

GPCRs G-protein coupled receptors

$N F K B$ Nuclear factor kappaB

Gpr G-protein receptor

SCFA short chain fatty acids

$T H$ t helper cell

${ }^{\mathrm{a}}$ Diet results in an altered microbiota

${ }^{\text {b}}$ Tryptophan metabolized by microbiota (e.g. lactobacilli) to indole-3 aldehyde and kynurenine (both AhR ligands) 
regulation of immune tolerance. The tryptophan metabolite kynurenine is an additional tryptophan-derived ligand of the AhR. Beside indole derivates, several other plant products, such as flavonoids and polyphenols, also bind to the AhR - however with lower affinity. The AhR therefore can be viewed as a major antiinflammatory factor that integrates dietary ("dietary pattern recognition receptors"), microbial, metabolic, and endogenous signals to alter the composition of the microbiota and elicit protective immune reactions (Table 1).

\section{Other anti-inflammatory components of cruciferous vegetables}

Sulforaphane (SFN), an isothiocyanate compound of cruciferous vegetables, protects from oxidative stress, inflammation and radiation injury. It inhibits LPS-induced monocyte adhesion via suppression of intercellular adhesion molecule-1 (ICAM-1) [23]. Furthermore, SCN also suppresses NF- $\kappa B$ activity in LPS-stimulated endothelial cells and these anti-inflammatory activities were dependent on intracellular glutathione levels. Such an inhibitory effect could also be observed in mouse peritoneal macrophages [24]. Interestingly, this anti-inflammatory effect was dependent on nuclear factor erythroid-2-related factor 2 (Nrf2) as it was not observed in Nrf2 (-/-) primary peritoneal macrophages. Nrf2 activates the transcription of more than 500 genes, most of which are protective and anti-inflammatory. Therefore, regulation of Nrf2 by isothiocyanates can be considered as an important aspect of its anti-inflammatory capacities. Nrf2 has been discussed to extend both healthspan and lifespan. Cruciferous vegetables can therefore be considered a diet with Nrf2-raising and therefore highly beneficial properties.

SFN is able to inhibit TNF- $\alpha$-induced NF- $k B$ activation through the inhibition of IкB $\alpha$ phosphorylation, IkB $\alpha$ degradation and p65 nuclear translocation [25]. This effect was paralleled by induction of apoptosis through activation of reactive oxygen species (ROS)-dependent caspase-3. Importantly, and this supports a relevant antiinflammatory role for SFN, it also suppresses vascular cell adhesion molecule (VCAM)-1 in LPS-stimulated endothelial cells [26]. In these experiments, SFN decreased the phosphorylation of extra-cellular signal-regulated kinase (ERK), JUN N-terminal kinase (JNK) and p38 mitogenactivated protein kinase (MAPK), all important inflammatory signaling cascades. In addition, SFN also affected TLR4 expression and suppressed MyD88, a key member of the signaling machinery of TLRs and the IL-1 pathway. Isothiocyanate suppresses LPS-induced synthesis of interferon-inducible protein-10 (IP-10) and phosphorylation of interferon regulatory factor 3 (IRF3) in RAW 264.7 cells [27]. Therefore, beneficial and anti-inflammatory effects of SFN might be mediated by various pathways including modulation of Toll-interleukin-1 receptor domain-containing adapter inducing interferon-beta (TRIF) signaling pathway of TLRs. SFN also shows antiatherosclerotic activities as it inhibits endothelial lipase (EL) activity in endothelial cells [28]. Endothelial lipase is a member of the triacylglycerol lipase family released during inflammation and has the capacity to decrease high-density lipoprotein levels. Therefore, suppression of EL by SCF could contribute to an important systemic anti-inflammatory profile exerted by cruciferous vegetables (Table 1).

Overall, these data suggest that SFN and above described mechanisms might contribute to anti-inflammatory properties of cruciferous vegetables. SFN also affects phagocytosis capacity of macrophages as it raises the phagocytic activity of RAW 264.7 murine macrophages [29]. Activation of phagocytosis remains an important mechanism to reduce and clear inflammatory insults. Recently it has also been shown that SFN inactivates macrophage migration inhibitory factor (MIF), an important inflammatory cytokine [30]. SFN is also protective in animal models of inflammation as it increases the survival of rats with hepatic failure as achieved after administration of D-galactosamine and LPS [31]. These effects were potentially achieved by its potent capability to suppress synthesis of proinflammatory cytokines such as TNF- $\alpha$ and Fas and ROS. SFN has also chemopreventive properties. An excessive expression of cyclooxygenase-2 (COX-2) links inflammation and cancer and SFN indeed suppresses COX-2 in human mammary epithelial cells after stimulation with 12-O-tetradecanoylphorbol-13-acetate (TPA) [32]. These effects were again mainly NF- $\kappa B$ - and ERK-mediated as demonstrated in earlier studies.

Berteroin (5-methylthiopentyl isothiocyanate) is another compound of cruciferous vegetables which is mainly present in cabbage, rucola salad leaves and mustard oil. Berteroin also decreases LPS-induced pro-inflammatory cytokines in RAW 264.7 macrophages. In the mouse ear, berteroin suppressed TPA-induced edema formation by down-regulating COX-2, NF-kB and ERK [33]. These authors suggested that this compound could be developed as local anti-inflammatory agent.

\section{Potential toxicological aspects of cruciferous vegetables}

Some earlier experimental studies have suggested that indole derivatives might exert detrimental effects including promotion of tumour development. High doses of $\mathrm{I} 3 \mathrm{C}$, probably never achieved after consumption in humans, exhibit a dose-dependent toxicity including a decrease in hepatic reduced glutathione and severe neurological toxicity in mice [34]. In another study, I3C after administration over 52 weeks showed a tendency for an increase of liver adenomas in rats after challenge with 
diethylnitrosamine and thyroid gland tumour incidence was increased significantly [35]. Indole derivatives promoted at high dietary levels aflatoxin B1-initiated hepatocarcinogenesis in rainbow trouts, an effect which was explained by the authors by the observed increase in estrogenic activities and induction of P450 isoenzymes [36]. Another study showed that I3C-treated rats developed fewer mammary adenocarcinomas but with a greater average weight per tumour per rat suggesting again that $\mathrm{I} 3 \mathrm{C}$ might negatively affect tumour growth [37]. I3C has also been shown to upregulate genes associated with signaling pathways for cell growth and proliferation suggesting that at least in this model I3C might result in a toxigenomic profile [38].

Human clinical data are not supportive of above experimental findings, although clinical trials focusing on the intake on cruciferous vegetables are rare. A recent meta-analysis suggested that consumption of cruciferous vegetables may reduce the risk of ovarian cancer [39]. In a large European cohort study, consumption of vegetables but not fruit was associated with a lower incidence of hepatocellular carcinoma [40]. A pooled analysis of three Italian case-control studies has demonstrated that a mediterranian diet reduces endometrial cancer risk [41]. A very large study from UK demonstrated that a diet enriched in vegetables and fresh fruits reduced both incidence of cardiovascular disease and cancer [42]. All these studies are in support of beneficial effects of vegetable consumption on human health, although more studies specifically addressing the effects of indole derivatives are needed.

\section{Conclusions}

There exists a crucial and exciting relationship between food, immunity, and the microbiota. Many dietary components affect these interactions. Dietary components exert either dominantly pro-or anti-inflammatory effects on the host. A healthy diet might contain a balanced mixture of pro- and anti-inflammatory dietary components. Knowledge in this field has increased dramatically in the last years. Key dietary players and their potential mechanisms have been characterized and how they might act harmful or beneficial on the host. Interventional studies have also demonstrated that dietary factors have strong effects on the microbiota and thereby might exert many immunomodulatory effects. However, it will be important to perform respective clinical studies in the next years to gain deeper mechanistic insights. Such studies could lead to development of functional foods, with beneficial and even therapeutic effects on the immune system. Therefore, food could in the future be used in clinical medicine to prevent and treat various diseases.

\section{Competing interests}

The author declares that he has no competing interests.

\section{Acknowledgement}

We gratefully acknowledge secretarial help by Mrs. Stephanie Federspiel-Kleinhans.

\section{Financial support}

Herbert Tilg is supported by the excellence initiative (Competence Centers for Excellent Technologies - COMET) of the Austrian Research Promotion Agency FFG: Research Center of Excellence in Vascular Ageing Tyrol, VASCage (K-Project Nr. 843536) funded by the BMVIT, BMWFW, the Wirtschaftsagentur Wien and the Standortagentur Tirol.

Received: 19 May 2015 Accepted: 20 July 2015

Published online: 09 November 2015

\section{References}

1. Veldhoen $\mathrm{M}$, Brucklacher-Waldert V. Dietary influences on intestinal immunity. Nat Rev Immunol. 2012;12:696-708.

2. Estruch R, Ros E, Salas-Salvado J, Covas Ml, Corella D, Aros F, et al. Primary prevention of cardiovascular disease with a Mediterranean diet. New Engl J Med. 2013;368:1279-90.

3. Tilg H, Moschen AR. Food, immunity, and the microbiome. Gastroenterology. 2015;148:1107-19.

4. Tilg H. Diet and intestinal immunity. New Engl J Med. 2012;366:181-3.

5. Li Y, Innocentin S, Withers DR, Roberts NA, Gallagher AR, Grigorieva EF, et al. Exogenous stimuli maintain intraepithelial lymphocytes via aryl hydrocarbon receptor activation. Cell. 2011;147:629-40.

6. Kiss EA, Vonarbourg C, Kopfmann S, Hobeika E, Finke D, Esser C, et al. Natural aryl hydrocarbon receptor ligands control organogenesis of intestinal lymphoid follicles. Science. 2011;334:1561-5.

7. Owyang C, Wu GD. The gut microbiome in health and disease. Gastroenterology. 2014;146:1433-6.

8. Moschen AR, Wieser V, Tilg H. Dietary Factors: Major Regulators of the Gut's Microbiota. Gut Liver. 2012;6:411-6.

9. Lee JY, Sohn KH, Rhee SH, Hwang D. Saturated fatty acids, but not unsaturated fatty acids, induce the expression of cyclooxygenase-2 mediated through Toll-like receptor 4. J Biol Chem. 2001;276:16683-9.

10. Huang S, Rutkowsky JM, Snodgrass RG, Ono-Moore KD, Schneider DA, Newman JW, et al. Saturated fatty acids activate TLR-mediated proinflammatory signaling pathways. J Lipid Res. 2012;53:2002-13.

11. Galli C, Calder PC. Effects of fat and fatty acid intake on inflammatory and immune responses: a critical review. Ann Nutr Metab. 2009;55:123-39.

12. Pendyala $\mathrm{S}$, Walker JM, Holt PR. A high-fat diet is associated with endotoxemia that originates from the gut. Gastroenterology. 2012;142:1100-1. e2.

13. Ding S, Chi MM, Scull BP, Rigby R, Schwerbrock NM, Magness S, et al. Highfat diet: bacteria interactions promote intestinal inflammation which precedes and correlates with obesity and insulin resistance in mouse. PLoS ONE. 2010;5:e12191.

14. Ghosh S, Molcan E, DeCoffe D, Dai C, Gibson DL. Diets rich in n-6 PUFA induce intestinal microbial dysbiosis in aged mice. Br J Nutr. 2013;1 10:515-23.

15. Jiang Y, Wu SH, Shu XO, Xiang YB, Ji BT, Milne GL, et al. Cruciferous vegetable intake is inversely correlated with circulating levels of proinflammatory markers in women. J Acad Nutr Diet. 2014;114:700-8. e2.

16. Wang X, Ota N, Manzanillo P, Kates L, Zavala-Solorio J, Eidenschenk C, et al. Interleukin-22 alleviates metabolic disorders and restores mucosal immunity in diabetes. Nature. 2014;514:237-41.

17. Julliard W, Fechner JH, Mezrich JD. The aryl hydrocarbon receptor meets immunology: friend or foe? A little of both. Front Immunol. 2014;5:458.

18. Mohammadi-Bardbori A, Bengtsson J, Rannug U, Rannug A, Wincent E. Quercetin, resveratrol, and curcumin are indirect activators of the aryl hydrocarbon receptor (AHR). Chem Res Toxicol. 2012;25:1878-84.

19. Yu TY, Pang WJ, Yang GS. 3,3'-Diindolylmethane increases bone mass by suppressing osteoclastic bone resorption in mice. J Pharmacol Sci. 2015;127:75-82.

20. Kim HW, Kim J, Kim J, Lee S, Choi BR, Han JS, et al. 3,3'-Diindolylmethane inhibits lipopolysaccharide-induced microglial hyperactivation and attenuates brain inflammation. Toxicol Sci. 2014;137:158-67.

21. Chang HP, Wang ML, Hsu CY, Liu ME, Chan MH, Chen YH. Suppression of inflammation-associated factors by indole-3-carbinol in mice fed high-fat diets and in isolated, co-cultured macrophages and adipocytes. Int J Obes. 2011;35:1530-8. 
22. Zelante T, lannitti RG, Cunha C, De Luca A, Giovannini G, Pieraccini G, et al. Tryptophan catabolites from microbiota engage aryl hydrocarbon receptor and balance mucosal reactivity via interleukin-22. Immunity. 2013;39:372-85.

23. Liu YC, Hsieh CW, Weng YC, Chuang SH, Hsieh CY, Wung BS. Sulforaphane inhibition of monocyte adhesion via the suppression of ICAM-1 and NF-kappaB is dependent upon glutathione depletion in endothelial cells. Vasc Pharmacol. 2008;48:54-61.

24. Lin W, Wu RT, Wu T, Khor TO, Wang H, Kong AN. Sulforaphane suppressed LPS-induced inflammation in mouse peritoneal macrophages through Nrf2 dependent pathway. Biochem Pharmacol. 2008;76:967-73.

25. Moon DO, Kim MO, Kang SH, Choi YH, Kim GY. Sulforaphane suppresses TNF-alpha-mediated activation of NF-kappaB and induces apoptosis through activation of reactive oxygen species-dependent caspase-3. Cancer Lett. 2009;274:132-42.

26. Shan Y, Lin N, Yang X, Tan J, Zhao R, Dong S, et al. Sulphoraphane inhibited the expressions of intercellular adhesion molecule-1 and vascular cell adhesion molecule-1 through MyD88-dependent toll-like receptor-4 pathway in cultured endothelial cells. Nutr Metab Cardiovasc Dis. 2012;22:215-22.

27. Park HJ, Kim SJ, Park SJ, Eom SH, Gu GJ, Kim SH, et al. Phenethyl isothiocyanate regulates inflammation through suppression of the TRIFdependent signaling pathway of Toll-like receptors. Life Sci. 2013;92:793-8.

28. Kivela AM, Makinen PI, Jyrkkanen HK, Mella-Aho E, Xia Y, Kansanen E, et al. Sulforaphane inhibits endothelial lipase expression through NF-kappaB in endothelial cells. Atherosclerosis. 2010;213:122-8.

29. Suganuma H, Fahey JW, Bryan KE, Healy ZR, Talalay P. Stimulation of phagocytosis by sulforaphane. Biochem Biophys Res Commun. 2011;405:146-51.

30. Crichlow GV, Fan C, Keeler C, Hodsdon M, Lolis EJ. Structural interactions dictate the kinetics of macrophage migration inhibitory factor inhibition by different cancer-preventive isothiocyanates. Biochemistry. 2012;51:7506-14.

31. Sayed RH, Khalil WK, Salem HA, Kenawy SA, El-Sayeh BM. Sulforaphane increases the survival rate in rats with fulminant hepatic failure induced by D-galactosamine and lipopolysaccharide. Nutr Res. 2014;34:982-9.

32. Kim HN, Kim DH, Kim EH, Lee MH, Kundu JK, Na HK, et al. Sulforaphane inhibits phorbol ester-stimulated IKK-NF-kappaB signaling and COX-2 expression in human mammary epithelial cells by targeting NF-kappaB activating kinase and ERK. Cancer Lett. 2014;351:41-9.

33. Jung YJ, Jung Jl, Cho HJ, Choi MS, Sung MK, Yu R, et al. Berteroin present in cruciferous vegetables exerts potent anti-inflammatory properties in murine macrophages and mouse skin. Int J Mol Sci. 2014;15:20686-705.

34. Shertzer HG, Sainsbury M. Intrinsic acute toxicity and hepatic enzyme inducing properties of the chemoprotectants indole-3-carbinol and 5,10dihydroindeno[1,2-b]indole in mice. Food Chem Toxicol. 1991;29:237-42.

35. Kim DJ, Han BS, Ahn B, Hasegawa R, Shirai T, Ito N, et al. Enhancement by indole-3-carbinol of liver and thyroid gland neoplastic development in a rat medium-term multiorgan carcinogenesis model. Carcinogenesis. 1997; 18:377-81.

36. Oganesian A, Hendricks JD, Pereira CB, Orner GA, Bailey GS, Williams DE. Potency of dietary indole-3-carbinol as a promoter of aflatoxin B1-initiated hepatocarcinogenesis: results from a 9000 animal tumor study. Carcinogenesis. 1999;20:453-8.

37. Malejka-Giganti D, Niehans GA, Reichert MA, Bliss RL. Post-initiation treatment of rats with indole-3-carbinol or beta-naphthoflavone does not suppress 7, 12-dimethylbenz[a]anthracene-induced mammary gland carcinogenesis. Cancer Lett. 2000;160:209-18.

38. Tilton SC, Givan SA, Pereira CB, Bailey GS, Williams DE. Toxicogenomic profiling of the hepatic tumor promoters indole-3-carbinol, 17beta-estradiol and beta-naphthoflavone in rainbow trout. Toxicol Sci. 2006;90:61-72.

39. $\mathrm{Hu}$ J, Hu Y, Hu Y, Zheng S. Intake of cruciferous vegetables is associated with reduced risk of ovarian cancer: a meta-analysis. Asia Pac J Clin Nutr. 2015;24:101-9.

40. Bamia C, Lagiou P, Jenab M, Aleksandrova K, Fedirko V, Trichopoulos D, et al. Fruit and vegetable consumption in relation to hepatocellular carcinoma in a multi-centre, European cohort study. Br J Cancer. 2015;112:1273-82

41. Filomeno M, Bosetti C, Bidoli E, Levi F, Serraino D, Montella M, et al. Mediterranean diet and risk of endometrial cancer: a pooled analysis of three italian case-control studies. Br J Cancer. 2015;112:1816-21.
42. Oyebode O, Gordon-Dseagu V, Walker A, Mindell JS. Fruit and vegetable consumption and all-cause, cancer and CVD mortality: analysis of Health Survey for England data. J Epidemiol Community Health. 2014;68:856-62.

43. Endres S, Ghorbani R, Kelley VE, Georgilis K, Lonnemann G, van der Meer $J W$, et al. The effect of dietary supplementation with n-3 polyunsaturated fatty acids on the synthesis of interleukin-1 and tumor necrosis factor by mononuclear cells. New Engl J Med. 1989;320:265-71.

\section{Submit your manuscript to a SpringerOpen ${ }^{\odot}$ journal and benefit from:}

- Convenient online submission

- Rigorous peer review

- Immediate publication on acceptance

- Open access: articles freely available online

- High visibility within the field

- Retaining the copyright to your article

Submit your next manuscript at springeropen.com 